\title{
Segmental dilatation of ileum in a young patient presenting with anemia
}

\author{
Neha Nischal, Deepak Balachandra'1, Anil Kumar Agarwal'1, Sunil Kumar Puri \\ Departments of Radiology and ${ }^{1}$ Gastro-intestinal Surgery, GB Pant Institute of Postgraduate Medical Education and \\ Research (GIPMER), New Delhi, India \\ Correspondence: Dr. Neha Nischal, Department of Radiology, GB Pant Hospital, 1, JLN Marg, New Delhi - 110002 , India. \\ E-mail: neha.nischal@gmail.com
}

\begin{abstract}
Segmental dilatation of ileum (SDI) is a less known uncommon entity with a confusing clinical scenario and no definite etiopathogenesis. The preoperative diagnosis is of exclusion. However, it has an excellent prognosis after surgery. We describe a case of a young patient who presented with anemia without any overt gastrointestinal (GI) bleed. Thorough radiological examinations were needed to reach the diagnosis of SDI which was confirmed postoperatively.
\end{abstract}

Key words: Barium studies; ileal dilatation; segmental dilatation

\section{Introduction}

Segmental dilatation of ileum (SDI) is a rare entity characterized by an idiopathic sharply demarcated segment of dilated ileum. It is difficult to diagnose clinically in view of absence of specific signs and symptoms. We describe the case of a young patient with SDI with emphasis on the clinico-radiological aspects of this entity.

\section{Case Report}

A 14-year-old male patient presented with complaints of vague abdominal pain and fullness on the right side of abdomen. He also had history of intermittent episodes of low-grade fever since 4 months. Clinical examination revealed mild distension on the right side of abdomen without a palpable mass. Laboratory investigations were normal except for anemia with $\mathrm{Hb}$ of $6.8 \mathrm{~g} \%$ (normal range 13.5-17 g\% for males). However, there was no history of any overt gastro-intestinal (GI) bleeding. Abdominal

\begin{tabular}{|c|c|}
\hline \multicolumn{2}{|c|}{ Access this article online } \\
\hline Quick Response Code: & \\
\hline \multirow{2}{*}{ 回触证回 } & $\begin{array}{l}\text { Website: } \\
\text { www.ijri.org }\end{array}$ \\
\hline & $\begin{array}{l}\text { DOI: } \\
\text { 10.4103/ijri.IJRI_403_17 }\end{array}$ \\
\hline
\end{tabular}

radiograph showed a large gas shadow in the right hypochondrium along with few enteroliths on the right side of abdomen [Figure 1]. An ultrasound of the abdomen revealed a large unilocular cystic lesion showing air-fluid level in the right lumbar and iliac region. Contrast-enhanced computed tomography (CECT) of the abdomen revealed similar imaging findings with the cyst showing air-fluid level and appearing to be in continuity with the ileum, suggesting dilated ileal loop [Figures 2 and 3]. However, no cause for obstruction could be found on CT. Barium meal follow-through (BMFT) examination was carried out later which proved that the cystic lesion was an aneurysmally dilated ileal loop with normal caliber of ileum proximal and distal to the dilated segment. The dilated segment showed normal peristalsis without evidence of any obstruction and was oriented along the long axis of the ileum [Figures 4 and 5].

Intraoperatively, there was large cystic dilatation of the mid-ileum, measuring about $15 \times 14 \mathrm{~cm}$, located about $90 \mathrm{~cm}$

This is an open access journal, and articles are distributed under the terms of the Creative Commons Attribution-NonCommercial-ShareAlike 4.0 License, which allows others to remix, tweak, and build upon the work non-commercially, as long as appropriate credit is given and the new creations are licensed under the identical terms.

For reprints contact: reprints@medknow.com

Cite this article as: Nischal N, Balachandra D, Agarwal AK, Puri SK. Segmental dilatation of ileum in a young patient presenting with anemia. Indian J Radiol Imaging 2018;28:369-72. 


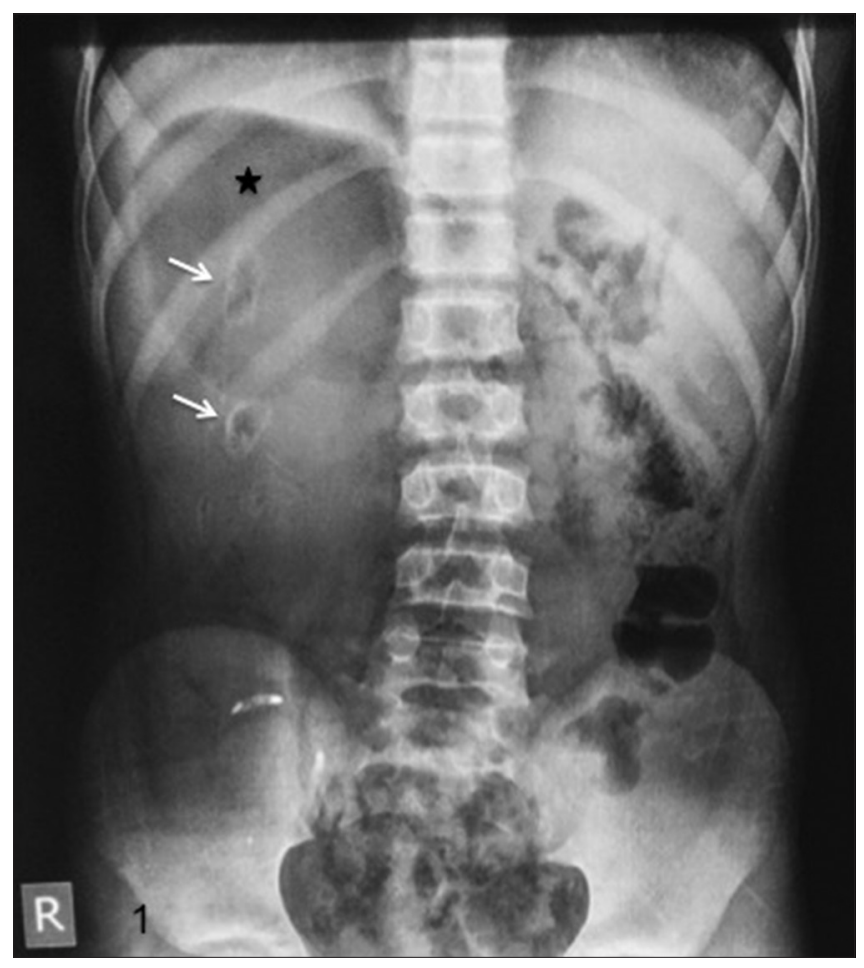

Figure 1: Erect radiograph of the abdomen showing a large air shadow in the right hypochondrium (star) with few enteroliths within the shadow (arrows)

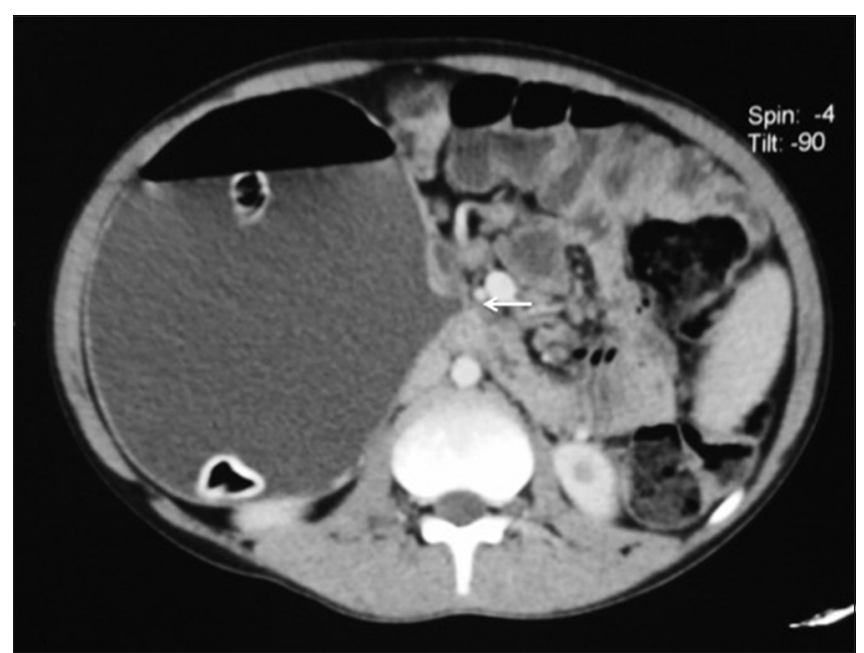

Figure 3: Axial CECT abdomen showing a large cystic lesion with air-fluid level and enteroliths. The efferent loop is marked with white arrow

proximal to the ileo-caecal junction causing postero-lateral displacement of the ascending colon [Figure 6]. The cut section showed normal mucosal folds in the dilated segment with $3 \times 2 \mathrm{~cm}$ irregular mucosa. Segmental resection with end-to-end anastomosis of the involved ileum was done. Postoperative course was uneventful. Biopsy showed normal bowel musculature and ganglion cells. An island of ectopic gastric mucosa was found corresponding to the irregular mucosa on gross examination. Final diagnosis of segmental dilatation of ileum (SDI) was made.

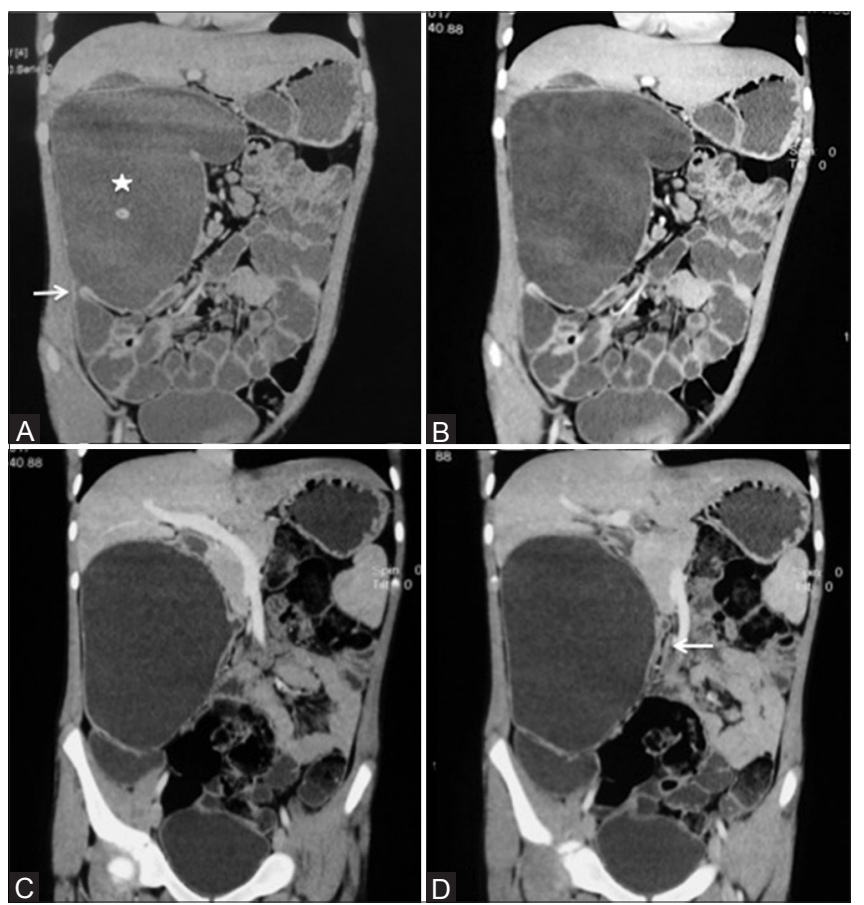

Figure 2 (A-D): Coronal CECT abdomen of the patient showing a hugely dilated ileal loop in the right sub-hepatic region (star in "A"). The afferent loop is shown with white arrow in "A." The efferent loop is collapsed and lying close to the dilated segment (arrow in "D")
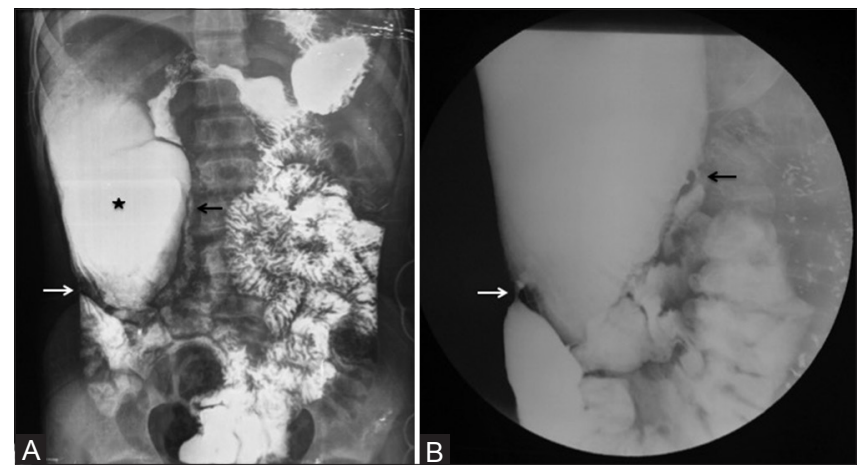

Figure 4 ( $A$ and $B$ ): Barium meal follow-through images ( $A$ and $B$ ) in the same patient showing passage of barium into the lesion (star in "A") with the orientation of the lesion along the long axis of bowel, confirming it to be segmentally dilated ileal loop. The afferent and efferent loops are marked with white and black arrows, respectively

\section{Discussion}

SDI, also known as segmental mega-ileum or ileal dysgenesis, is an unusual congenital dilatation of a segment of bowel which most commonly involves ileum. ${ }^{[1]}$ The entity was initially reported by Swenson and Rathauser in 1959. Their case involved the colon. Since then, fewer than 200 cases have been published worldwide. ${ }^{[2]}$ They also proposed the following criteria for diagnosing this entity: (1) limited dilatation of bowel with threefold or fourfold increased size; (2) sharp conversion between dilated and normal intestine without an obvious obstruction; (3) the 


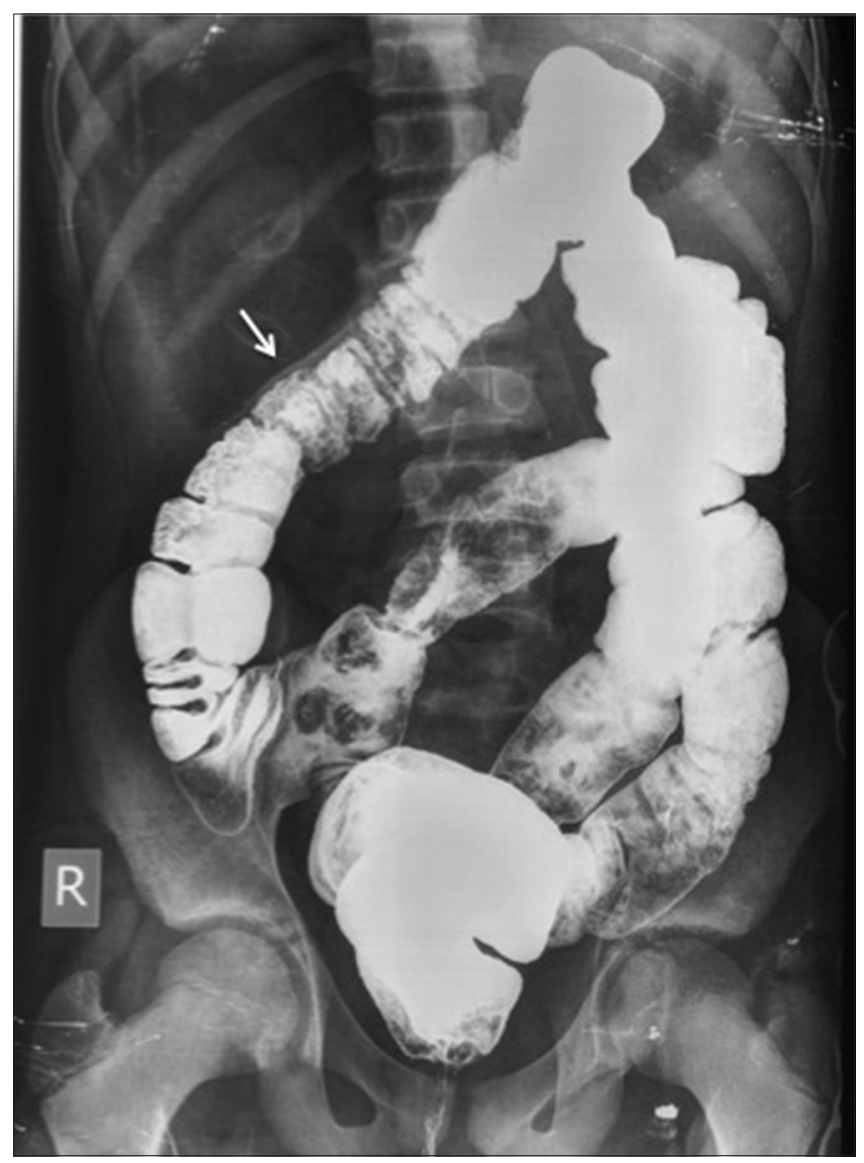

Figure 5: Delayed image of the BMFT study showing barium in the large bowel which is normal in caliber. The ascending colon and hepatic flexure are displaced toward left by the dilated air-filled loop (arrow). Note that there is no residual barium within the dilated ileal segment

presence of normal neuronal plexus in submucosa and muscularispropria; (4) clinical presentation of obstruction or sub-obstruction; and (5) recovery after resection of the dilated segment. ${ }^{[3,4]}$

Presentation is commonly in infancy or childhood and symptoms vary with age at presentation. In the neonatal period, obstruction is the commonest feature owing to the aperistaltic bowel segment. However, later in life, the patient may present with GI bleeding, anemia, abdominal pain, or may be asymptomatic. ${ }^{[2,4]}$ The cause of overt or occult GI bleeding is attributed to the presence of ulcers and/or ectopic gastric mucosa within the dilated segment. A high proportion of these cases are reported to have other GI tract anomalies including omphalocele, malrotation, and Meckel's diverticulum. ${ }^{[1-4,6,7]}$ A case of SDI with anterior thoracolumbar meningocoele and bilateral undescended testes has also been described in literature ${ }^{[7]}$ In this report, the patient had vague abdominal pain with asymptomatic anemia, without any associated GI anomalies.

The etiopathogenesis is still debatable. However, most authors postulate an extrinsic source of compression in

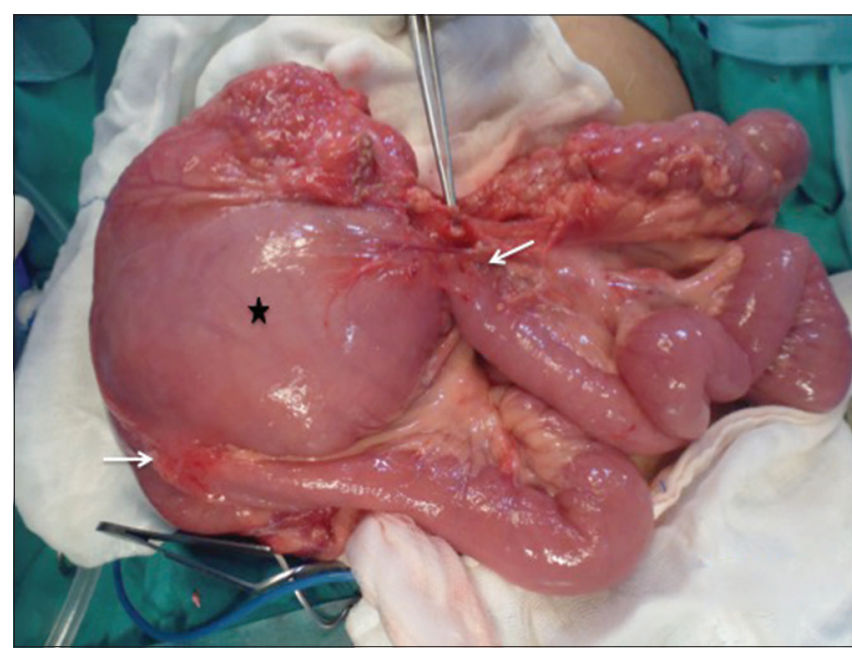

Figure 6: Intraoperative finding of an aneurysmally dilated ileal loop (star) with proximal and distal normal appearing loops (arrows)

utero including a band in an exomphalos, a vitelline band arising from Meckel's diverticulum, or vitelline vessels that involute postnatally. Another theory suggests that during the period of physiologic herniation, the abdominal wall may act as an obstructing lesion. These hypotheses remain unresolved as of now. The possibility of local neuropathy or myopathy has also not been proved conclusively by histo-pathological studies. ${ }^{[1,2,6,8]}$

Radiological examinations are quite contributory to the diagnosis. Abdominal radiograph usually reveals dilated bowel loop on the right side with or without air-fluid levels, which may suggest possibility of cecal volvulus in the setting of intestinal obstruction. Ultrasound reveals a large cystic lesion, which may mimic a mesenteric cyst or duplication cyst of the small bowel. Barium studies disclose the nature of the lesion as being a dilated segment along the long axis of bowel, as opposed to Meckel's diverticulum or other small bowel diverticuli which show anti-mesenteric or mesenteric orientation. Other differentials may include aneurysmal dilatation in lymphoma or cavitating neoplasms, but CT usually suffices in ruling out these causes ${ }^{[3,6,8]} \mathrm{CT}$ enteroclysis is reported to be even more sensitive in diagnosing this lesion accurately by achieving adequate distension of bowel. ${ }^{[6]}$

Exclusion of Hirschsprung disease is imperative before making the diagnosis of SDI where there is normal neuronal plexus within the dilated segment. ${ }^{[3]}$

Many authors have also labeled this entity as a giant Meckel's diverticulum. However, there does exist some differences between the two, most important being the anti-mesenteric orientation of the Meckel's diverticulum as opposed to SDI, which is oriented along the long axis of bowel. Heterotopic gastric or pancreatic mucosa may be found in both the entities and should not be the criteria for diagnosing giant Meckel's diverticulum. ${ }^{[9,10]}$ 
The treatment is resection of the involved segment with end-to-end bowel anastomosis which has an extremely favorable outcome for the patient. ${ }^{[1-5,7]}$

\section{Conclusion}

In the light of pauci-clinical manifestations, imaging has paramount role in reaching the correct preoperative diagnosis of SDI. High index of suspicion should be kept in young patients with unexplained anemia or GI bleed showing cystic dilatation of ileum. We also propose to change the nomenclature of giant Meckel's diverticulum because of the inherent differences between the two entities.

\section{Declaration of patient consent}

The authors certify that they have obtained all appropriate patient consent forms. In the form the patient(s) has/have given his/her/their consent for his/her/their images and other clinical information to be reported in the journal. The patients understand that their names and initials will not be published and due efforts will be made to conceal their identity, but anonymity cannot be guaranteed.

Financial support and sponsorship

Nil.

\section{Conflicts of Interest}

There are no conflicts of interest.

\section{References}

1. Sam CJ. Segmental ileal dilatation in a child. Trop Gastroenterol 2011;32:221-3.

2. Paradiso FV, Coletta R, Olivieri C, Briganti V, Oriolo L, Fabbri R, et al. Antenatal ultrasonographic features associated with segmental small bowel dilatation: An unusual neonatal condition mimicking congenital small bowel obstruction. Pediatr Neonatol 2013;54:339-43.

3. Wei $\mathrm{CH}$, Sheu JC. Concomitant segmental intestinal dilatation and omphalocele. Formos J Surg 2011;44:168-70.

4. Khemakhem R, Riazulhaq M, Elhassan EO. Segmental dilatation of intestine presenting as partial intestinal obstruction in a child. APSP J Case Rep 2014;5:19.

5. Sjolin S, Thoren L. Segmental dilatation of the small intestine. Arch Dis Child 1962;37:422-4.

6. Lee RKL, Hung EHY, Leung JHY, Tsang KWK. Idiopathic localised dilatation of the ileum: Computed tomography enteroclysis. Hong Kong J Radiol 2014;17:124-8.

7. Raj P, Sarin YK. Segmental dilatation of ileum associated with anterior thoracolumbar meningomyelocele and bilateral undescended testes. APSP J Case Rep 2015;6:5.

8. Javors BR, Gold RP, Ghahremani GG, Radin DR, Cho KC, Maglinte DD, et al. Idiopathic localized dilatation of the ileum in adults: Findings on barium studies. Am J Roentgenol 1995;164:87-90.

9. Akbulut S, Yagmur Y. Giant Meckel's diverticulum: An exceptional cause of intestinal obstruction. World J Gastrointest Surg 2014;6:47-50.

10. Nunes Q, Hotouras A, Tiwari S, Sheth A. Gangrene due to axial torsion of a giant Meckel's diverticulum containing multiple stones in the lumen: A case report. Cases J 2009;2:7141. 